\title{
Differentially expressed serum proteins in children with or without asthma as determined using isobaric tags for relative and absolute quantitation proteomics
}

\author{
Ming Li ${ }^{\text {Equal first author, } 1}$, Mingzhu Wu ${ }^{\text {Equal first author, } 2}{ }^{2}$ Ying Qin ${ }^{3}$, Huaqing Liu ${ }^{1}$, Chengcheng Tu ${ }^{2}$, Bing Shen ${ }^{3}$, Xiaohong Xu \\ Corresp., 4 , HongBo Chen ${ }^{\text {Corresp. } 2}$ \\ ${ }^{1}$ Department of Neonatology, Maternal and Child Health Hospital, the Affiliated Hospital of Anhui Medical University, Hefei, Anhui, China \\ 2 Department of Obstetrics and Gynecology, Maternal and Child Health Hospital, the Affiliated Hospital of Anhui Medical University, Hefei, Anhui, China \\ ${ }^{3}$ School of Basic Medicine, Anhui Medical University, Hefei, Anhui, China \\ 4 Department of Clinical Laboratory, Maternal and Child Health Hospital, the Affiliated Hospital of Anhui Medical University, Hefei, Anhui, China \\ Corresponding Authors: Xiaohong Xu, HongBo Chen \\ Email address: xuxiao1234@163.com, chenhongbo@ahmu.edu.cn
}

Background. Although asthma is one of the most common chronic, noncommunicable diseases worldwide, the pathogenesis of childhood asthma is not yet clear. Genetic factors and environmental factors may lead to airway immune-inflammation responses and an imbalance of airway nerve regulation. The aim of the present study was to determine which serum proteins are differentially expressed between children with or without asthma and to ascertain the potential roles that these differentially expressed proteins (DEPs) may play in the pathogenesis of childhood asthma.

Methods. Serum samples derived from four children with asthma and four children without asthma were collected. The DEPs were identified by using isobaric tags for relative and absolute quantitation (iTRAQ) combined with liquid chromatography tandem mass spectrometry (LC-MS/MS) analyses. Using biological information technology, including Gene Ontology (GO), Kyoto Encyclopedia of Genes and Genomes (KEGG), and Cluster of Orthologous Groups of Proteins (COG) databases and analyses, we determined the biological processes associated with these DEPs. Key protein glucose-6-phosphate dehydrogenase (G6PD) was verified by enzyme linked immunosorbent assay (ELISA).

Results. We found 46 DEPs in serum samples of children with asthma vs. children without asthma. Among these DEPs, 12 proteins were significantly ( $>1.5$ fold change) upregulated and 34 proteins were downregulated. The results of GO analyses showed that the DEPs were mainly involved in binding, the immune system, or responding to stimuli or were part of a cellular anatomical entity. In the KEGG signaling pathway analysis, most of the downregulated DEPs were associated with cardiomyopathy, phagosomes, viral infections, and regulation of the actin cytoskeleton. The results of a COG analysis showed that the DEPs were primarily involved in signal transduction mechanisms and posttranslational modifications. These DEPs were associated with and may play important roles in the immune response, the inflammatory response, extracellular matrix degradation, and the nervous system. The downregulated of G6PD in the asthma group was confirmed using ELISA experiment.

Conclusion. After bioinformatics analyses, we found numerous DEPs that may play important roles in the pathogenesis of childhood asthma. Those proteins may be novel biomarkers of childhood asthma and may provide new clues for the early clinical diagnosis and treatment of childhood asthma. 
1 Differentially Expressed Serum Proteins in Children with or without Asthma as Determined

2 Using Isobaric Tags for Relative and Absolute Quantitation Proteomics

3 Ming Li ${ }^{1, \#}$, Mingzhu $\mathrm{Wu}^{2, \#}$, Ying Qin ${ }^{3}$, Huaqing Liu ${ }^{1}$, Chengcheng $\mathrm{Tu}^{2}$, Bing $\mathrm{Shen}^{3}$, Xiaohong

$4 \mathrm{Xu}^{4, *}$, Hongbo $\mathrm{Chen}^{2, *}$

5

$6{ }^{1}$ Department of Neonatology, Maternity and Child Health Hospital, the Affiliated Hospital of

7 Anhui Medical University, Hefei, Anhui, China

8 2Department of Obstetrics and Gynecology, Maternal and Child Health Hospital, the Affiliated

9 Hospital of Anhui Medical University, Hefei, Anhui, China

$10{ }^{3}$ School of Basic Medicine, Anhui Medical University, Hefei, Anhui, China

$11{ }^{4}$ Department of Clinical Laboratory, Maternal and Child Health Hospital, the Affiliated Hospital

12 of Anhui Medical University, Hefei, Anhui, China

14 \#, These authors contributed equally to this work

$16 *$,Corresponding author contact information

17 Corresponding Author:

18 Hongbo Chen, Ph.D.

Department of Obstetrics and Gynecology, Maternity and Child Health Hospital, the Affiliated

20 Hospital of Anhui Medical University, 15 Yimin Road, Hefei, Anhui 230001, China.

21 Tel: +86-18056076983, Email address: chenhongbo@ahmu.edu.cn.

22

23 Xiaohong $\mathrm{Xu}$, Prof. 
24 Department of Clinical Laboratory, Maternity and Child Health Hospital, the Affiliated Hospital 25 of Anhui Medical University, 15 Yimin Road, Hefei, Anhui 230001, China.

26 Tel: +86-13855110036, Email address: xuxiao1234@163.com 


\section{Abstract}

28 Background. Although asthma is one of the most common chronic, noncommunicable diseases worldwide, the pathogenesis of childhood asthma is not yet clear. Genetic factors and environmental factors may lead to airway immune-inflammation responses and an imbalance of airway nerve regulation. The aim of the present study was to determine which serum proteins are differentially expressed between children with or without asthma and to ascertain the potential roles that these differentially expressed proteins (DEPs) may play in the pathogenesis of childhood

34 asthma.

Methods. Serum samples derived from four children with asthma and four children without asthma were collected. The DEPs were identified by using isobaric tags for relative and absolute quantitation (iTRAQ) combined with liquid chromatography tandem mass spectrometry (LCMS/MS) analyses. Using biological information technology, including Gene Ontology (GO), Kyoto Encyclopedia of Genes and Genomes (KEGG), and Cluster of Orthologous Groups of Proteins (COG) databases and analyses, we determined the biological processes associated with these DEPs. Key protein glucose-6-phosphate dehydrogenase (G6PD) was verified by enzyme linked immunosorbent assay (ELISA).

Results. We found 46 DEPs in serum samples of children with asthma vs. children without asthma. Among these DEPs, 12 proteins were significantly ( $>1.5$ fold change) upregulated and 34 proteins were downregulated. The results of GO analyses showed that the DEPs were mainly involved in binding, the immune system, or responding to stimuli or were part of a cellular anatomical entity. In the KEGG signaling pathway analysis, most of the downregulated DEPs were associated with cardiomyopathy, phagosomes, viral infections, and regulation of the actin cytoskeleton. The results of a COG analysis showed that the DEPs were primarily involved in signal transduction 
50 mechanisms and posttranslational modifications. These DEPs were associated with and may play

51 important roles in the immune response, the inflammatory response, extracellular matrix

52 degradation, and the nervous system. The downregulated of G6PD in the asthma group was

53 confirmed using ELISA experiment.

54 Conclusion. After bioinformatics analyses, we found numerous DEPs that may play important

55 roles in the pathogenesis of childhood asthma. Those proteins may be novel biomarkers of

56 childhood asthma and may provide new clues for the early clinical diagnosis and treatment of

57 childhood asthma.

58

59 
60

61 Asthma is one of the most common chronic noncommunicable diseases. According to reports from

62 different countries, the current global prevalence rate of asthma is $1 \%-18 \%$ (Neelamegan et al.

63 2016), threatening approximately 334 million people worldwide. Asthma is characterized by

64 airway hyperresponsiveness, reversible airway obstruction, and chronic airway inflammation.

65 Symptoms are often recurrent and worsen with time (Papi et al. 2018). Asthma presents its highest

66 incidence in childhood and affects children's quality of life. Serious cases may lead to death, which

67 brings great economic burdens to families and to health systems (Pincheira et al. 2020). Therefore,

68 identifying pathological mechanisms and finding new therapeutic targets for asthma are urgent.

69

70

Despite a worldwide presence and economic burden, the pathogenesis of childhood asthma is still unclear. It is currently thought that under the influence of genetic and environmental factors, the mechanisms underlying asthma include inflammatory cells, cytokines, and inflammatory mediators acting on the airway to cause airway inflammation and remodeling, and the imbalance

74 of airway nerve regulation and abnormal structure and function of airway smooth muscle lead to 75 airway hyperresponsiveness and induce asthma (Demenais et al. 2018; Morales \& Duffy 2019; 76 Papi et al. 2018). There are two types of asthma. The first is called eosinophilic asthma and is 77 characterized by an imbalance in the helper $\mathrm{T}(\mathrm{Th})$ cell $\mathrm{Th} 1 / \mathrm{Th} 2$ ratio. The second type is called 78 non-eosinophilic asthma and is mainly neutrophilic asthma that is controlled by Th17 (Lambrecht \& Hammad 2015). Children with asthma typically present with eosinophilic asthma and allergy, 80 which easily leads to airway remodeling (Hamsten et al. 2016). 
82 In asthma, some specific proteins produced by tissue cells may be secreted into the circulation.

83 Thus, proteomics may be a useful approach to detect and quantitate such serum proteins and to

84 determine whether they are differentially expressed in asthma and may be potential therapeutic

85 targets. For example, by combining affinity proteomics with the human protein atlas, Hamsten et

86 al. (Hamsten et al. 2016) discovered that selective chemokine (C-C motif) ligand 5 (CCL5),

87 hematopoietic prostaglandin D synthase (HPGDS), and neuropeptide S receptor 1 (NPSR1) were

88 involved in inflammatory reactions and affected the onset of childhood asthma. In addition,

89 Suojalehto et al. (Suojalehto et al. 2015) identified differentially regulated proteins by using two-

90 dimensional differential gel electrophoresis and mass spectrometry. They determined that fatty

91 acid binding protein 5 (FABP5) was increased in the sputum of patients with allergic asthma and

92 showed the relationship of this protein with airway remodeling and inflammation (Suojalehto et

93 al. 2015). However, among the currently available proteomics methods, isobaric tags for relative

94 and absolute quantitation (iTRAQ) is considered to be most effective (Moulder et al. 2018).

95 Therefore, to gain mechanistic insights into the pathogenesis of childhood asthma, the present

96 study used iTRAQ technology combined with liquid chromatography tandem mass spectrometry

97 (LC-MS/MS) to analyze the protein composition and expression levels in serum samples obtain

98 from children with or without asthma. Using bioinformatics analyses, we aimed to determine key

99 proteins that may be (1) used as biological markers or (2) part of critical signaling pathways

100 involved in the development of asthma or (3) useful in determining the prognosis of children with

101 asthma.

102

103 Materials \& Methods

104 Experimental design 
105 The study proceeded according to the flowchart shown in Fig. 1.

106

107 Clinical information and serum sample collection

108 From September to October 2019, serum samples were collected from eight children who had

109 received no treatment but who had been admitted to the Maternal and Child Health Hospital of

110 Anhui Medical University Affiliated Hospital. No child included in the study had received a

111 diagnosis of an immune disease, chronic kidney disease, or other disease affecting serum proteins.

112 The included samples were collected from four children with asthma (experimental group, all

113 samples were belongs to eosinophilic asthma, and obtained after the diagnosis immediately but

114 before drug treatment) and four children without asthma (control group). All clinical diagnoses

115 followed the 2019 Global Initiative for Asthma guidelines. The experiment was approved by the

116 Medical Ethics Committee of Anhui Medical University (approval number 20200284), and the

117 parents or guardians of all participants signed informed consent forms.

118

119 On the morning of the second day after the children were admitted to the hospital without drug 120 treatment, blood samples $(4 \mathrm{~mL})$ were collected, placed at room temperature $\left(22-25^{\circ} \mathrm{C}\right)$ in the

121 dark for $1 \mathrm{~h}$, and centrifuged at $3000 \mathrm{rpm}$ at $4{ }^{\circ} \mathrm{C}$ for $15 \mathrm{~min}$. The supernatant was transferred to a 122 new tube with a pipette and stored at $-80^{\circ} \mathrm{C}$ until it was used in an experiment.

123

124 Protein extraction and quality control

125 A ProteoExtract albumin/IgG removal kit (Merck \& Co.) was used to extract the serum samples.

126 The total amount of protein extracted from each one serum sample was more than $400 \mu \mathrm{g}$. The

127 protein bands were clear, complete and uniform. The protein was not degraded, and the total 
128 amount of protein in each sample was able to be used to do two or more experiments. The protein

129 solution was prepared, and the Bradford Protein Assay working solution was added. Sodium

130 dodecyl sulfate-polyacrylamide gel electrophoresis (SDS-PAGE) and Coomassie blue staining

131 were performed to evaluate the sample quality after detecting the total amount of protein.

133 Labeling after enzymatic hydrolysis of proteins

134 We used iTRAQ techniques to label the peptide segments after enzymolysis (Sandberg et al. 2012).

135 After protein quantification, $60 \mu \mathrm{g}$ of the protein solution was placed in a centrifuge tube and $5 \mu \mathrm{L}$

136 dithiothreitol solution was added. The mixture remained at $37{ }^{\circ} \mathrm{C}$ for $1 \mathrm{~h}$. Next, $20 \mu \mathrm{L}$ of

137 iodoacetamide solution was added, and the mixture was placed at room temperature in the dark for

$1381 \mathrm{~h}$. The samples were centrifuged, and the collected supernatant was discarded. The precipitated 139 sediment was twice treated with $100 \mu \mathrm{L}$ of uric acid buffer (8 M urea, $100 \mathrm{mM}$ Tris-HCl; $\mathrm{pH}$ 8.0).

140 The samples were washed with $\mathrm{NH}_{4} \mathrm{HCO}_{3}(50 \mathrm{mM}, 100 \mu \mathrm{L})$ three times, and trypsin (the ratio of 141 protein to enzyme 50:1) was added to the sample in an ultrafiltration tube. Enzymolysis was 142 performed on the samples at $37^{\circ} \mathrm{C}$ for $12-16 \mathrm{~h}$. Finally, the samples were labeled and desalted 143 using a C18 cartridge.

144

145 LC-MS/MS analysis

146 We identified differentially expressed proteins (DEPs) between the two groups by using an LC147 MS/MS Spectrum system (Sandberg et al. 2012). After re-dissolving the labeled samples in $40 \mu \mathrm{L}$ 148 of $0.1 \%$ formic acid aqueous solution, we analyzed them using nano-LC-MS/MS. The mobile 149 phases were phase A ( $2 \%$ acetonitrile $/ 0.1 \%$ formic acid $/ 98 \%$ water $)$ and phase B $(80 \%$ 150 acetonitrile $/ 0.08 \%$ formic acid $/ 20 \%$ water). The column was equilibrated with $95 \%$ phase A liquid. 
151 The gradient from phase B was adjusted as follows: 0-80 min, linear increase from $0 \%$ to $40 \%$;

$15280-80.1 \mathrm{~min}$, increased to $95 \% ; 80.1-85 \mathrm{~min}$, maintained at $95 \% ; 85-88 \mathrm{~min}$, decreased to $6 \%$.

153 The separated samples were analyzed using mass spectrometry with a Q Exactive HF-X Mass

154 Spectrometer (Thermo Fisher Scientific). Proteome Discover software, version 2.2.0.388, was

155 used to search the Uniport Human database in FASTA format. Protein fold changes (FC) of at

156 least 1.5 were obtained, and $P<0.05$ was considered a statistically significant difference. A FC

$157>1.5$ represented upregulated proteins and a $\mathrm{FC}<0.667$ represented downregulated proteins; a FC

158 between 0.667 and 1.5 indicated no obvious change in protein expression between the two groups

159 (Morrissey et al. 2013; Oliveira et al. 2020; Rawat et al. 2016).

160

161 Gene Ontology (GO), Kyoto Encyclopedia of Genes and Genomes (KEGG), and Cluster of 162 Orthologous Groups of Proteins (COG) Signaling Pathway Analyses

$163 \mathrm{GO}$ is a database that can be applied to various species to define and describe the functions of 164 genes and proteins (Fang et al. 2019). The GO database is often used to clarify the roles of eukaryotic genes and proteins in cells. GO is useful for comprehensively describing the attributes of genes and gene products in organisms. GO consists of three domains (Liu et al. 2020; Wang et al. 2019a; Xing et al. 2020): (1) the cellular component domain contains the descriptions of proteins related to cell composition, which may be a subcellular structure (e.g., endoplasmic reticulum or nucleus) or a protein production component (e.g., ribosome, or proteasome); (2) the molecular function domain contains descriptions of all proteins related to molecular functions, 171 such as biological activities and operations performed by specific gene products (i.e., molecules or complexes); (3) the biological process domain contains descriptions of all proteins related to

173 biological processes, that is, a series of events in which molecular functions cooperate with one 
174 another. The GO function annotation result refers to the statistical number of DEPs detected

175 between the two groups of serum samples in the three GO domains. The GO functional

176 significance enrichment analysis provides the GO functional terms significantly enriched with the

177 DEPs compared with all identified proteins, thus determining which biological functions the DEPs

178 are significantly related to. The GO terms can explain the role of eukaryotic genes and proteins in

179 cells, thus comprehensively describing the attributes of genes and gene products in organisms (Cai 180 et al. 2015).

181

182 KEGG is a group of databases used to connect a series of genes in the genome with a molecular 183 interaction network in cells to identify biological functions at the genomic and molecular levels.

184 KEGG contains the signaling pathways of multiple cell processes, such as metabolism, membrane 185 conversion, signal transduction, and the cell cycle (Kanehisa \& Goto 2000). The results of a KEGG 186 analysis provides insights into the higher biological functions of cells. KEGG analysis can provide 187 the most important biochemical metabolic pathways and signal transduction pathways involved in 188 protein (Yang et al. 2018). COG annotates the functions of homologous proteins and includes both 189 the COG database (clusters of homologous proteins from prokaryotes) and the KOG database 190 (clusters of homologous proteins in eukaryotes) (Tatusov et al. 1997). COG database can provide 191 the function of differential proteins (Wu et al. 2019). Briefly, using BLASTP (BLAST version 192 2.2.30, http://blast.ncbi.nlm.nih.gov/Blast.cgi), the sequence of protein set was compared with the 193 COG database (the expected value of BLAST alignment parameter was set to 1 e-5), and the 194 corresponding COG number of protein was obtained. The corresponding function description and 195 function classification of protein were acquired. 
197

198

199

200

201

202

203

204

205

206

207

208

209

210

21

212

21

214

215

216

217

218

219

\section{Enzyme linked immunosorbent assay (ELISA)}

Glucose-6-phosphate dehydrogenase (G6PD), the downregulated protein in the asthma group, was verified by ELISA kit. The experiment was performed according to the kit instructions. Blood samples were collected from children with or without asthma.

\section{Statistical Analysis}

Two-tailed Mann-Whitney test was performed with SigmaPlot software. The chi-square test by Fisher's exact test was used to compare the categorical variables (only gender data). Values are expressed as means \pm SEM. A value of $P<0.05$ was considered statistically significant.

\section{Results}

\section{Participants}

In the participants of LC-MS/MS experiments, no significant difference in age (asthma : control, means $\pm \mathrm{SD}, 4.0 \pm 1.0$ vs. $3.3 \pm 0.6$ years; $\mathrm{n}=8, P=0.5$, gender (asthma : control, 3 males and 1 female vs. 2 males and 2 females, $P=0.5$ ) or body mass index (asthma : control, means $\pm \mathrm{SD}$, $19.1 \pm 0.6$ vs. $17.9 \pm 0.9 \mathrm{~kg} / \mathrm{m} 2 ; \mathrm{n}=8, P=0.3$ ) was detected between the experimental group (children with asthma) and the control group (children with trauma but no infection or asthma).

\section{SDS-PAGE}

Serum samples obtained from children with or without asthma were separated using SDS-PAGE.

Total protein from eight samples was effectively separated without protein degradation within the molecular weight range of 15-220 kDa. The protein levels were sufficient to be used in subsequent experiments (Fig. 2). 


\section{LC-MS/MS analysis and identification of DEPS}

222 223

224 225 226 227 228 229 230 231 232 233 234 235 236 237 238 239 240 241

LC-MS/MS is a powerful tool that enables identification of proteins in a mixed sample. In total, 103 proteins were identified in the serum of children with or without asthma, of which 46 DEPs were detected. As shown in Table 1, 12 DEPs were upregulated and 34 DEPs were downregulated. We plotted the magnitude of FC $\left(\log _{2} \mathrm{FC}\right)$ on the $\mathrm{x}$-axis and the statistical significance of that change ( $-\log _{10}$ of the $P$ value) on the y-axis to obtain a volcano plot (Fig. $\left.3 \mathrm{~A}\right)$. The cluster analysis for the expression of the DEPs clearly indicated that the expression patterns between children with or without asthma differed and that the protein expression in each group was clustered together (Fig. 3B). These results suggested that there was a significant difference in the levels of proteins expressed in the serum of children with or without asthma.

\section{GO functional annotation and enrichment analysis}

The GO functional annotation results of the DEPs between children with or without asthma are shown in Fig. 4. For the cellular component domain, 37 DEPs were mainly concentrated in the cellular anatomical term, among which the top 3 upregulated proteins were IGKV2-40, IGHV374, and IGKV1-27. For the molecular function domain, the role of 38 DEPs was mainly in binding, among which the top 3 upregulated proteins were IGKV2-40, IGHV3-74, and IGKV1-27. For the biological process domain, the DEPS were primarily involved in cellular processes (31 DEPs) and responding to stimuli (30 DEPs), with the top 3 upregulated proteins being IGKV2-40, IGHV374, and V1-19. 
242 Significant enrichment analysis of the GO function refers to a rough understanding of the

243 biological processes in which DEPs are enriched based on the simple annotation of genes, which

244 increases the reliability of research focused on determining pathogenesis. By analyzing the GO

245 functional significance enrichment map of the DEPs between children with or without asthma (Fig.

246 5), it was found that the detected DEPs play important roles as cellular anatomical entities and in

247 binding, cellular processes, and responding to stimuli.

248

249 KEGG metabolic pathway analysis

250 In organisms, different proteins perform their biological functions in coordination with one 251 another. Analyses based on metabolic pathways are helpful to further understand the biological 252 functions of the DEPs. KEGG is the main public database used to analyze such pathways, and 253 analyses using KEGG can determine the most important biochemical metabolic pathways and

254 255 signal transduction pathways in which the DEGs participate. Here, KEGG functional annotation analysis was carried out on the DEPs identified in the serum samples from children with or without asthma. The results showed that KEGG pathways annotated with the DEPs included dilated cardiomyopathy (DCM), hypertrophic cardiomyopathy (HCM), phagosomes, regulation of the actin cytoskeleton, focal adhesions, and metabolic pathways (Fig. 6). The downregulated DEPs associated with DCM/HCM were ITGB1, ITGA2, ACTB, and CACNA2D1. The downregulated DEPs associated with phagosomes were ITGB1, ITGA2, ACTB, and MPO. The downregulated DEPs associated with regulation of the actin cytoskeleton were ITGB1, ITGA2, ACTB, and PFN1. The downregulated DEPs associated with focal adhesions were ITGB1, ITGA2, TNXB, and ACTB. The downregulated DEPs associated with metabolic pathways were AOC3, ALPL, PKM2, and G6PD. The pathway enrichment analysis is the same as the GO functional enrichment analysis, 
265 which uses the KEGG pathway as a unit and applies hypergeometric testing to determine the 266 pathways significantly enriched with DEPs compared with all identified proteins. The most 267 important biochemical metabolic pathways and signal transduction pathways of DEPs can be 268 determined by pathway enrichment analysis (Fig. 7). The present analysis showed that most of the 269 detected downregulated proteins were concentrated in metabolic pathways such as focal adhesion, 270 MAPK signaling pathway, platelet activation and Rap1 signaling pathway..

271

\section{COG protein functional analysis}

273 COG is a database for orthologous classification of proteins. It is assumed that the group of 274 proteins constituting each COG are all derived from the same ancestral protein and are divided 275 into orthologs and paralogs. Orthologs refer to proteins evolved from vertical families (speciation) 276 of different species and typically retain the same functions as the original proteins. Paralogs refer 277 to proteins derived from gene replication in certain species that may evolve new functions related 278 to the original functions. The DEPs between children with or without asthma were analyzed using 279 the COG database (Fig. 8). We predicted the possible functions of these protein, and generated the 280 functional classification statistics. The results showed that the functions of these DEPs were 281 mainly concentrated in signal transduction mechanisms; posttranslational modification, protein 282 turnover, and chaperones; amino acid transport and metabolism; cytoskeleton; and extracellular 283 structures.

\section{G6PD protein concentration in serum}

286 To confirm the DEP in the LC-MS/MS experiment, we used an ELISA experiment and choose a 287 key protein G6PD, which is decreased in asthma reported by studies from other groups (Hirasawa 
288 et al. 2003). Our data also showed a significant reduction in asthma group compared to control 289 group (Fig. 9). Therefore, the result is consistent with the data from LC-MS/MS experiment.

290

291 Discussion

292 The pathological mechanisms underlying asthma are still not clear; however, the application of 293 proteomics may be valuable for finding some new clues. Recently, several studies also used 294 proteomics to try to find biomarkers in asthma. Gharib et al. utilized shortgun proteomics method 295 to identify protein expression pattern in adult induced sputum samples (Gharib et al. 2011); 296 Bhowmik et al. used quantitative label-free liquid chromatography-tandem mass spectrometry 297 method to find that apolipoprotein E (ApoE) is significantly downregulated but interleukin 33 (IL298 33) is significantly upregulated in serum samples from adult atopic asthma compared to healthy 299 control (Bhowmik et al. 2019); Moreover, helped by method of iTRAQ combined with LCMS/MS, Liu et al. reported DEPs in serum samples from children patients with controlled, partly 301 controlled, or uncontrolled childhood asthma (Liu et al. 2017). However, in our study, we used iTRAQ method to identify the DEPs in the serum of children with asthma vs. those without asthma. Asthma is an allergic response, that is, an individual's own immune system overreacts. In a previous study, it was found that when the human airway is exposed to invading pathogens, the congenital immune process is rapidly induced (Lebold et al. 2016). The congenital immune system 306 is the first line of defense in the human immune system (Yin et al. 2020). When the body is infected 307 by foreign agents, inflammatory reactions will occur first (Kimbrell \& Beutler 2001). These 308 reactions can produce a variety of chemical factors, including cytokines (Kzhyshkowska \& Bugert 309 2016), to recruit immune cells (e.g., macrophages, neutrophils) to infected or inflamed tissues to 310 kill or inhibit the growth of pathogens through phagocytosis and other actions to prevent the spread 
311 of infection. Inflammatory reactions can also promote the healing of injured tissues. Another

312 defensive reaction to invading pathogens is the complement system, which helps or complements

313 the antibody itself to remove or to label antigenic substances to control pathogens (Hato \& Pierre

314 2015). Complement component 1s (C1S) is involved in the complement and coagulation cascade

315 in the metabolic pathway of the KEGG database. In our GO analysis, we found that several proteins

316 that are involved in complement activation (namely, IGHV3-74, IGLC7, and IGKV1-16) were

317 differentially expressed between children with or without asthma. These mass spectrometry results

318 are consistent with results from a previous study (Hato \& Pierre 2015). However, we also detected

319 DEPs that have not been reported in previous studies on childhood asthma, including IGKV2-40,

320 IGHV3-74, IGKV1-27, IGKV1-16, CTSG, HSPA1A, SERPINB1, LTF, IGLC7, CTSG,

321 ADAMTS13, NCAM2, TNXB, ACTB, and CNTN1. These findings may indicate that when

322 infection causes a rapid congenital immune response and airway inflammation, airway remodeling

323 will be induced, leading to the onset or exacerbation of childhood asthma (Krusche et al. 2019;

324 Johnston et al. 1995). In addition, inflammation is divided into local manifestations and systemic

325 reactions. Small trauma may cause local inflammation. When local lesions are serious, especially

326 when pathogenic microorganisms spread in the body, obvious systemic reactions often occur. The

327 children in the control group had only minor local trauma which did not develop systemic

328 inflammation. However, the inflammatory reaction in asthmatic children prefers a systemic 329 inflammation.

330

331 By analyzing DEPs through GO terms or KEGG pathway analysis, we found that the upregulated

332 proteins IGKV2-40, IGHV3-74, IGKV1-27, and IGKV1-16 were involved in the innate immune

333 process. Most of the downregulated proteins were involved in the congenital immune system and 
334 neutrophil degranulation, including CTSG, MPO, IFNa2, HSPA1A, SERPINB1, C1S, and LTF.

335 Among them, IFNa2 is produced after the body recognizes many pathogens and damage-related

336 molecules released by infected or dead cells and is a key component of the innate immune response

337 (Paul et al. 2015). HSPA1A can exert immune functions through fusion with the membrane,

338 endocytosis, autophagy, and interaction with ligands (Bilog et al. 2019; Oliverio et al. 2018;

339 Sangaphunchai et al. 2020). SERPINB1 is a neutrophil elastase inhibitor that plays an important

340 role in regulating cell activity, inflammatory response, and cell migration (Torriglia et al. 2017).

341 Therefore, our findings provided several new potential protein targets in the immune system for

342 treatment of children with asthma.

344 Among the upregulated proteins, the GO terms or KEGG pathways analysis showed that the DEPs 345 participate in immunoglobulin production (IGKV2-40, IGKV1-27, and IGKV1-16), receptor346 mediated endocytosis (IGKV2-40, IGLC7, and IGKV1-16), phagocytosis, recognition and 347 engulfment (IGHV3-74, IGLC7), and complement activation (IGHV3-74, IGLC7, and IGKV1348 16). The downregulated proteins are involved in extracellular matrix degradation (CTSG, TNXB, 349 ELANE, and COL10A1), metabolism of various substances (CTSG, ADAMTS13, DPP4, G6PD, 350 APOB, ACAN, AOC3, and LRP1), and cell adhesion and focal adhesion (NCAM2, TNXB, 351 ACTB, CNTN1, ITGB1, and ELANE). During airway remodeling, epithelial cell exfoliation, 352 goblet cell proliferation, vascular proliferation, extracellular matrix deposition, and hypertrophy 353 of smooth muscle cells are involved. When the expression levels of ELANE are downregulated, 354 the degradation of the extracellular matrix decreases, which will aggravate airway remodeling 355 (Linden et al. 2005). Other group (Hirasawa et al. 2003) found that MAPK and activated protein 356 kinases are related to viral replication. Lacking G6PD will increase the products of cellular reactive 
357 oxygen species, which strengthens those kinase pathways to facilitate viral replication, thus

358 inducing or aggravating asthma. AOC3 is commonly referred to as vascular adhesion protein-1

359 (VAP-1) and is expressed in lymphocyte endothelial interactions. As a new marker of 360 myofibroblasts, AOC3 may play a role in pulmonary fibrosis and thus induce asthma (Hsia et al.

361 2016). The imbalance in the expression levels of LRP1 in fibroblasts of healing tissues may lead

362 to unlimited expansion of contractile fibroblasts, thus causing or aggravating pulmonary fibrosis

363 and participating in the pathogenesis of asthma (Schnieder et al. 2019). Neural cell adhesion

364 molecule 2 (NCAM2) protein has been shown to reduce inflammation in Alzheimer's disease

365 (Rasmussen et al. 2018). CNTN1, which is also a cell adhesion protein, can promote the invasion

366 of prostate cancer cells, enhance Akt activation, and reduce the expression of epithelial cadherin

367 in cancer cells (Yan et al. 2016). Some studies have found that DPP4 plays an important role in

368 angiogenesis and growth, immune response, cell proliferation, fibrin degradation, cytokine

369 production, signal transduction, and other physiological and pathological processes of the body

370 (Liu et al. 2015; Mark 2005; Ohnuma et al. 2015; Ta et al. 2010). In addition, DPP4 is widely

371 distributed in lung tissue and participates in pulmonary inflammation and the formation of

372 pulmonary surfactant (Mentlein 1999; Schmiedl et al. 2014). We speculate that when a pathogenic

373 infection exists in the lungs of children, the downregulation of DPP4 will lead to an immune

374 imbalance and inflammatory cascade reaction to affect the formation of lung substances, thus

375 inducing asthma. However, our findings and speculation should be confirmed in future studies.

376

377 Other DEPs detected in our KEGG analysis also play important roles in cellular physiological and

378 pathological activities and may be part of mechanisms underlying childhood asthma. Among the

379 upregulated proteins, APP participates in the following pathways: neuron growth, adhesion, axon 
380 generation, cell migration, regulation of protein movement, cell apoptosis, and combination with

381 extracellular matrix components. Some studies have also found that APP plays an important role

382 in the pathogenesis of Alzheimer's disease and is overexpressed in cancer cells, such as in

383 nasopharyngeal carcinoma (Li et al. 2019a; Li et al. 2019b). Another upregulated protein, PIP, a

384 pathway annotated in KEGG, has a regulatory effect on natural immune cells. Some studies have

385 found that PIP can specifically degrade fibronectin to help hosts resist infection and play a role in 386 the migration, adhesion, and invasion of cancer cells (Ihedioha et al. 2018; Kitano et al. 2006;

387 Wang et al. 2019b).

388

389 Among the downregulated proteins detected through our GO term or KEGG pathway analysis,

390 CACNA2D1 is involved in the macrophage CCR5 pathway; ITGB1 is involved in the adaptive 391 immune process, cell adhesion, blood-brain barrier, immune cell migration, and cytoskeleton 392 signaling process; and LTF is involved in amyloid fiber formation. Thus, through KEGG analyses, 393 we have provided insights into the potential mechanisms involved in the development of childhood 394 asthma.

395

\section{Conclusions}

397 We used iTRAQ combined with LC-MS/MS technologies to identify DEPs in serum samples 398 between children with or without asthma. The present study provided additional evidence 399 consistent with the role of the inflammation-immune mechanism in the pathogenesis of childhood 400 asthma, and offered new potential biomarkers for childhood asthma that may be helpful for its 401 early diagnosis. The analysis of signaling pathways provided numerous key proteins that may be 
402 involved in the development of childhood asthma and that may be new targets in the study of 403 asthma in future.

404

405 Funding Sources

406 This work was supported by grants from the Anhui Province Science and Technology Innovation 407 Project Demonstration Project (No. 201707d08050003), and the Anhui Province Key Research 408 and Development Project (No. 201904a07020032), and the National Natural Science Foundation 409 of China (grant Nos. 81570403, 81371284 and U1732157).

410

411 Competing interests: The authors declare that they have no conflict of interest.

412

413 References

414 Bhowmik M, Majumdar S, Dasgupta A, Bhattacharya S, and Saha S. 2019. Pilot-Scale Study Of 415 Human Plasma Proteomics Identifies ApoE And IL33 As Markers In Atopic Asthma. 416 Journal of Asthma and Allergy:273-283. 10.2147/JAA.S211569

417 Bilog A, Smulders L, Oliverio R, Labanieh C, Zapanta J, Stahelin R, and Nikolaidis N. 2019. 418 Membrane Localization of HspA1A, a Stress Inducible 70-kDa Heat-Shock Protein, 419 Depends on Its Interaction with Intracellular Phosphatidylserine. Biomolecules 9. $420 \quad 10.3390 /$ biom9040152

421 Cai A, Qi S, Su Z, Shen H, Yang Y, He L, and Dai Y. 2015. Quantitative Proteomic Analysis of 422 Peripheral Blood Mononuclear Cells in Ankylosing Spondylitis by iTRAQ. Clinical \& 423 Translational Science 8:579-583. 10.1111/cts.12265

424 Demenais F, Margaritte-Jeannin P, Barnes K, Cookson W, Altmüller J, Ang W, Barr R, Beaty T, 
425

426

427

428

429

430

431

432

433

434

435

436

437

438

439

440

441

442

443

444

445

446

447

Becker A, and Beilby J. 2018. Multiancestry association study identifies new asthma risk loci that colocalize with immune-cell enhancer marks. 50:42-53.

Fang Y, Wang P, Xia L, Bai S, Shen Y, Li Q, Wang Y, Zhu J, Du J, and Shen B. 2019. Aberrantly hydroxymethylated differentially expressed genes and the associated protein pathways in osteoarthritis. PeerJ 7:e6425. 10.7717/peerj.6425

Gharib S, Nguyen E, Lai Y, Plampin J, Goodlett D, and Hallstrand T. 2011. Induced Sputum Proteome in Health and Asthma. J Allergy Clin Immunol 128(6):1176-1184. 10.1016/j.jaci.2011.07.053

Hamsten C, Haggmark A, Grundstrom J, Mikus M, Lindskog C, Konradsen JR, Eklund A, Pershagen G, Wickman M, Grunewald J, Melen E, Hedlin G, Nilsson P, and van Hage M. 2016. Protein profiles of CCL5, HPGDS, and NPSR1 in plasma reveal association with childhood asthma. Allergy 71:1357-1361. 10.1111/all.12927

Hato T, and Pierre C. 2015. How the Innate Immune System Senses Trouble and Causes Trouble. Clin J Am Soc Nephrol 10:1459-1469. 10.2215/CJN.04680514

Hirasawa K, Kim A, Han H, Han J, Jun H, and Yoon J. 2003. Effect of p38 mitogen-activated protein kinase on the replication of encephalomyocarditis virus. J Virol 77:5649-5656. 10.1128/jvi.77.10.5649-5656.2003

Hsia L, Ashley N, Ouaret D, Wang L, Wilding J, and Bodmer W. 2016. Myofibroblasts are distinguished from activated skin fibroblasts by the expression of AOC3 and other associated markers. Proc Natl Acad Sci U S A 113:E2162-2171. 10.1073/pnas.1603534113

Ihedioha O, Blanchard A, Balhara J, Okwor I, Jia P, Uzonna J, and Myal Y. 2018. The human breast cancer-associated protein, the prolactin-inducible protein (PIP), regulates intracellular signaling events and cytokine production by macrophages. Immunol Res 
449 Johnston S, Pattemore P, Sanderson G, Smith S, Lampe F, Josephs L, Symington P, O'Toole S, H

450

451

452

453

454

455

456

457

458

459

460

461

462

463

464

465

466

467

468

469

470

Myint S, Tyrrell D, and Holgate S. 1995. Community study ofrole ofviral infections in exacerbations ofasthma in 9-11 year old children.

Kanehisa M, and Goto S. 2000. KEGG: Kyoto Encyclopedia of Genes and Genomes. Nucleic Acids 28:27-30.

Kimbrell D, and Beutler B. 2001. The Evolution and Genetics of Innate Immunity. Nature Reviews Genetics.

Kitano T, Tian W, Umetsu K, Yuasa I, Yamazaki K, Saitou N, and Osawa M. 2006. Origin and evolution of gene for prolactin-induced protein. Gene 383:64-70. 10.1016/j.gene.2006.07.014

Krusche K, Basse S, and Schaub B. 2019. Role of early life immune regulation in asthma development. Semin Immunopathol. 10.1007/s00281-019-00774-Z

Kzhyshkowska J, and Bugert P. 2016. Innate Immune System for Diagnostics and Therapy: Progress in Fundamental Knowledge and Clinical Application. Transfus Med Hemother 43:63-64. 10.1159/000445488

Lambrecht B, and Hammad H. 2015. The immunology of asthma. Nat Immunol 16:45-56. 10.1038/ni.3049

Lebold K, Jacoby D, and Drake M. 2016. Toll-Like Receptor 7-Targeted Therapy in Respiratory Disease. Transfus Med Hemother 43:114-119. 10.1159/000445324

Li N, Liu K, Qiu Y, Zhang H, Nakanishi H, and Qing H. 2019a. Mutations of beta-amyloid precursor protein alter the consequence of Alzheimer's disease pathogenesis. Neural Regen Res 14:658-665. 10.4103/1673-5374.247469 
471 Li X, Meng H, Li K, Yang X, Zhu X, Li L, Liang Z, Pan X, Zeng F, and Qu S. 2019b. Amyloid 472 Beta (A4) Precursor Protein: A Potential Biomarker for Recurrent Nasopharyngeal 473 Carcinoma. Cancer Manag Res 11:10651-10656. 10.2147/CMAR.S218030

474 Linden A, Laan M, and Anderson G. 2005. Neutrophils, interleukin-17A and lung disease. Eur 475 Respir J 25:159-172. 10.1183/09031936.04.00032904

476

477

478

479

480

481

482

483

484

485

486

487

488

489

490

491

492

493

Liu G, Chen C, Kong N, Tian R, Li Y, Li Z, Wang K, and Yang P. 2020. Identification of potential miRNA biomarkers for traumatic osteonecrosis of femoral head. J Cell Physiol. $10.1002 /$ jcp. 29467

Liu J, Wang Y, Wang Y, Zhu J, and Dao F. 2017. Screening Serum Differential Proteins for Childhood Asthma at Different Control Levels by Isobaric Tags for Relative and Absolute Quantification-based Proteomic Technology. Acta Academiae Medicinae Sinicae 39:817826. 10.3881/j.issn.1000-503X.2017.06.014

Liu P, Chen C, Wang C, Wu Y, Hsu C, Lee C, Huang L, Yu J, Chang Y, Wu C, and Yu C. 2015. In-depth proteomic analysis of six types of exudative pleural effusions for nonsmall cell lung cancer biomarker discovery. Mol Cell Proteomics 14:917-932. 10.1074/mcp.M114.045914

Mark D. 2005. Dipeptidyl peptidase IV and related enzymes in cell biology and liver disorders. Clinical Science 108:277-292

Mentlein R. 1999. Dipeptidyl-peptidase IV (CD26)-role in the inactivation of regulatory peptides. Regulatory Peptides 85(1):9-24.

Morales E, and Duffy D. 2019. Genetics and Gene-Environment Interactions in Childhood and Adult Onset Asthma. Front Pediatr 7:499. 10.3389/fped.2019.00499

Morrissey B, O' Shea C, Armstrong J, Rooney C, Staunton L, Sheehan M, Shannon A, and 
494

495

496

497

498

499

500

501

502

503

504

505

506

507

508

509

510

511 Oliverio R, Nguyen P, Kdeiss B, Ord S, Daniels A, and Nikolaidis N. 2018. Functional 512

Pennington S. 2013. Development of a label-free LC-MS/MS strategy to approach the identification of candidate protein biomarkers of disease recurrence in prostate cancer patients in a clinical trial of combined hormone and radiation therapy. 10.1002/prca.201300004

Moulder R, Bhosale S, Goodlett D, and Lahesmaa R. 2018. Analysis of the plasma proteome using iTRAQ and TMT-based Isobaric labeling. Mass Spectrom Rev 37:583-606. $10.1002 / \operatorname{mas} .21550$

Neelamegan R, Saka V, Tamilarasu K, Rajaram M, Selvarajan S, and Chandrasekaran A. 2016. Clinical Utility of Fractional exhaled Nitric Oxide (FeNO) as a Biomarker to Predict Severity of Disease and Response to Inhaled Corticosteroid (ICS) in Asthma Patients. $J$ Clin Diagn Res 10:FC01-FC06. 10.7860/JCDR/2016/20656.8950

Ohnuma K, Hatano R, and Morimoto C. 2015. DPP4 in anti-tumor immunity: going beyond the enzyme. Nat Immunol 16:791-792. 10.1038/ni.3210

Oliveira T, Lacerda J, Leite G, Dias M, Mendes M, Kassab P, Silva C, Juliano M, and Forones N. 2020. Label-free peptide quantification coupled with in silico mapping of proteases for identification of potential serum biomarkers in gastric adenocarcinoma patients. Clinical Biochemistry. 10.1016/j.clinbiochem.2020.02.010

characterization of natural variants found on the major stress inducible $70-\mathrm{kDa}$ heat shock gene, HSPA1A, in humans. Biochem Biophys Res Commun 506:799-804. 10.1016/j.bbrc.2018.10.148

Papi A, Brightling C, Pedersen S, and Reddel H. 2018. Asthma. The Lancet 391:783-800. $10.1016 / \mathrm{s} 0140-6736(17) 33311-1$ 
517 Paul F, Pellegrini S, and Uzé G. 2015. IFNA2: The prototypic human alpha interferon. Gene 518 567:132-137. 10.1016/j.gene.2015.04.087

519 Pincheira M, Bacharier L, and Castro-Rodriguez J. 2020. Efficacy of Macrolides on Acute Asthma 520 or Wheezing Exacerbations in Children with Recurrent Wheezing: A Systematic Review

521 and Meta-analysis. Paediatr Drugs. 10.1007/s40272-019-00371-5

522 Rasmussen K, Falkesgaard M, Winther M, Roed N, Quistgaard C, Teisen M, Edslev S, Petersen 523 D, Aljubouri A, Christensen C, Thulstrup P, Leggio L, Teilum K, and Walmod P. 2018. NCAM2 Fibronectin type-III domains form a rigid structure that binds and activates the Fibroblast Growth Factor Receptor. Sci Rep 8:8957. 10.1038/s41598-018-27089-7

526

527

528

529

530

531

532

533

534

535

536

537

538

539

Behere P, Balhara A, Vashisth R, Singh I, Dang A, Kaushik J, Mohanty T, and Mohanty A. 2016. Identification of potential protein biomarkers for early detection of pregnancy in cow urine using 2D DIGE and label free quantitation. Clinical Proteomics 13:15. $10.1186 / \mathrm{s} 12014-016-9116-\mathrm{y}$

Sandberg A, Lindell G, Kallstrom B, Branca R, Danielsson K, Dahlberg M, Larson B, Forshed J, and Lehtio J. 2012. Tumor proteomics by multivariate analysis on individual pathway data for characterization of vulvar cancer phenotypes. Molecular \& Cellular Proteomics 11:M112 016998. 10.1074/mcp.M112.016998

Sangaphunchai P, Todd I, and Fairclough L. 2020. Extracellular Vesicles and Asthma: a review of the literature. Clin Exp Allergy. 10.1111/cea.13562

Schmiedl A, Grützner D, Hoffmann T, von Hörsten S, and Stephan M. 2014. DPP4 inhibitors increase differentially the expression of surfactant proteins in Fischer 344 rats. Acta Physiol (Oxf) 212:248-261. 10.1111/apha.12350

Peer) reviewing PDF | (2020:03:47037:2:0:NEW 22 Aug 2020) 
540 Schnieder J, Mamazhakypov A, Birnhuber A, Wilhelm J, Kwapiszewska G, Ruppert C, Markart 541 P, Wujak L, Rubio K, Barreto G, Schaefer L, and Wygrecka M. 2019. Loss of LRP1 542 promotes acquisition of contractile-myofibroblast phenotype and release of active TGF543 beta1 from ECM stores. Matrix Biol. 10.1016/j.matbio.2019.12.001

544 Suojalehto H, Kinaret P, Kilpeläinen M, Toskala E, Ahonen N, Wolff H, Alenius H, and Puustinen 545

546

547

548

549

550 A. 2015. Level of Fatty Acid Binding Protein 5 (FABP5) Is Increased in Sputum of Allergic Asthmatics and Links to Airway Remodeling and Inflammation. PLoS One 10:e127003.

\section{1}

552 10.1371/journal.pone.0127003

Ta N, Li Y, Schuyler C, Lopes-Virella M, and Huang Y. 2010. DPP-4 (CD26) inhibitor alogliptin inhibits TLR4-mediated ERK activation and ERK-dependent MMP-1 expression by U937 histiocytes. Atherosclerosis 213:429-435. 10.1016/j.atherosclerosis.2010.08.064

Tatusov R, Koonin E, and Lipman D. 1997. A Genomic Perspective on Protein Families. Science 278:631-637.

Torriglia A, Martin E, and Jaadane I. 2017. The hidden side of SERPINB1/Leukocyte Elastase Inhibitor. Semin Cell Dev Biol 62:178-186. 10.1016/j.semcdb.2016.07.010

555

556

557

558

559

560

561 562

Wang L, Zhang G, Kang F, Zhang L, and Zhang Y. 2019a. hsa_circ0021347 as a Potential Target Regulated by B7-H3 in Modulating the Malignant Characteristics of Osteosarcoma. Biomed Res Int 2019:9301989. 10.1155/2019/9301989

Wang Q, Zhi Y, Ren W, Li S, Quan X, Xing X, Gao L, Wang Y, and Zhi K. 2019b. Suppression of OSCC malignancy by oral glands derived-PIP identified by iTRAQ combined with 2D LC-MS/MS. J Cell Physiol. 10.1002/jcp.28180

Wu W, Lin X, Wang C, Ke J, Wang L, and Liu H. 2019. Transcriptome of white shrimp Litopenaeus vannamei induced with rapamycin reveals the role of autophagy in shrimp 
564 Xing X, Jiang Y, Wang H, Zhang Y, Niu T, Qu Y, Wang C, Wang H, and Liu K. 2020. 565 Identification of novel differentially expressed genes in retinas of STZ-induced long-term 566 diabetic rats through RNA sequencing. Mol Genet Genomic Med:e1115. 567 $10.1002 / \mathrm{mgg} 3.1115$

568 569

570

571

572 Yang Y, Fu X, Qu W, Xiao Y, and Shen H. 2018. MiRGOFS: a GO-based functional similarity 573 574 575 576 577

578 Major P, Wood G, Peng H, and Tang D. 2016. Neural Cell Adhesion Protein CNTN1 Promotes the Metastatic Progression of Prostate Cancer. Cancer Res 76:1603-1614. 10.1158/0008-5472.CAN-15-1898 measurement for miRNAs, with applications to the prediction of miRNA subcellular localization and miRNA-disease association. Bioinformatics. 10.1093/bioinformatics/bty343

Yin X, Langer S, Zhang Z, Herbert K, Yoh S, König R, and Chanda S. 2020. Sensor SensibilityHIV-1 and the Innate Immune Response. Cells 9. 10.3390/cells9010254 
Figure 1

Flowchart of the experimental design.

COG represents Clusters of Orthologous Groups; GO, Gene Ontology; KEGG, Kyoto Encyclopedia of Genes and Genomes; iTRAQ, isobaric tags for relative and absolute quantitation; and SDS-PAGE, sodium dodecyl sulfate-polyacrylamide gel electrophoresis. 
Collect serum samples from:

Children with asthma (experimental group)

Children without asthma

(control group)

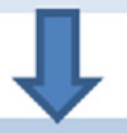

Separate and determine quality of serum proteins using SDS-PAGE

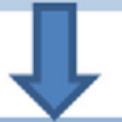

Conduct quantitative proteomics analysis using iTRAQ

Perform bioinformatic analyses using $\mathrm{GO}$, KEGG, and COG

Determine potential mechanisms underlying the pathogenesis of childhood asthma 


\section{Figure 2}

Protein separation and quality control using sodium dodecyl sulfate-polyacrylamide gel electrophoresis.

In this representative western blot, lane B1 is the original serum from a control child without asthma and $\mathrm{M}$ is the marker of B1. Lanes 1-4 are low-abundance proteins obtained from serum samples of children in the asthmatic group; and lanes 5-8 are low-abundance proteins obtained from serum samples of children in the control group.

$\begin{array}{llllllllll}\text { B1 } & \text { M } & 1 & 2 & 3 & 4 & 5 & 6 & 7 & 8\end{array}$

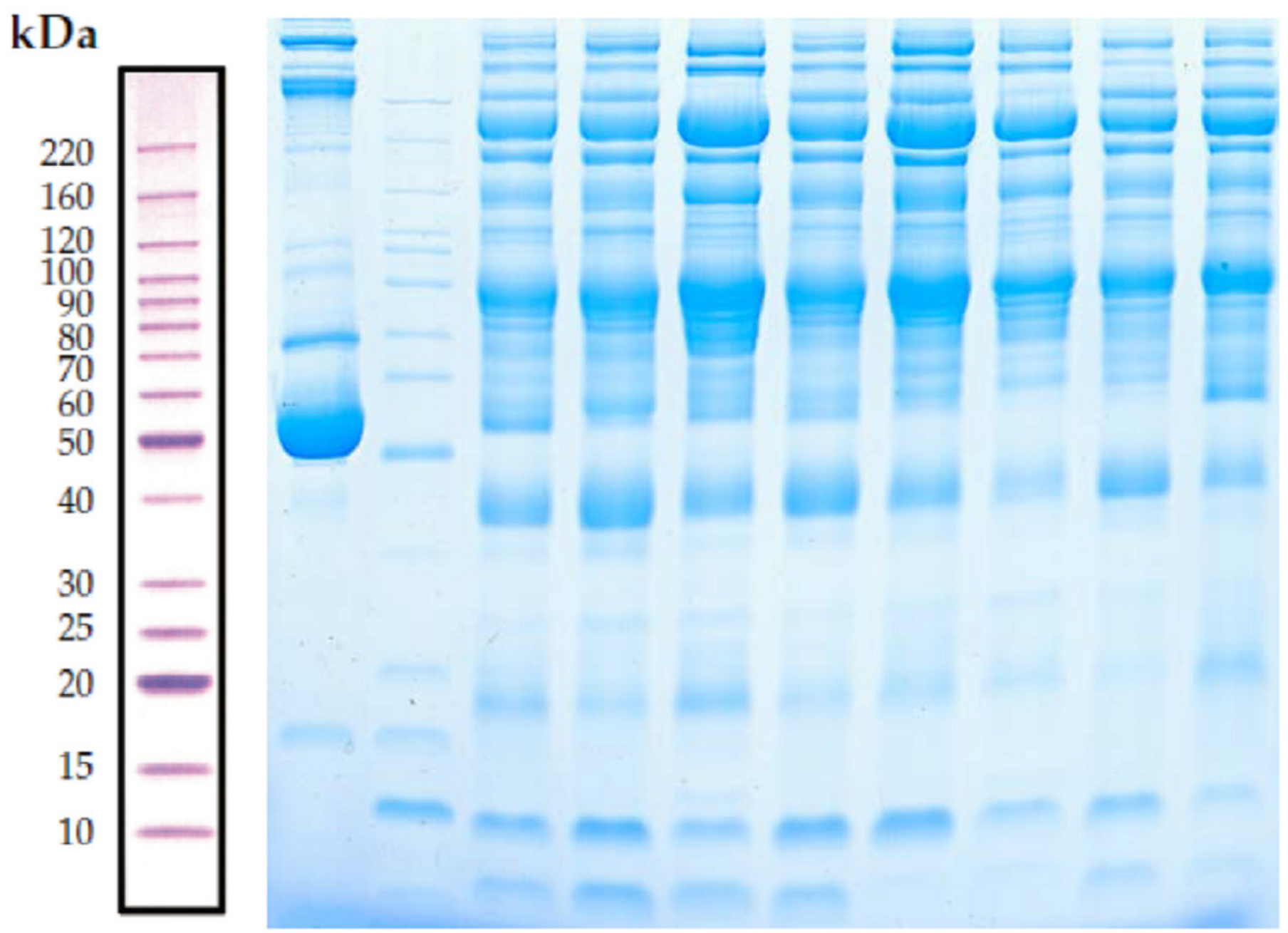




\section{Figure 3}

Volcano plot and heatmap of differentially expressed proteins (DEPs) in children with or without asthma.

Figure 3. Volcano plot and heatmap of differentially expressed proteins (DEPs) in children with or without asthma. (A) In this volcano plot, yellow dots represent proteins with a significant fold change $(F C)>1.5$; blue-green dots, proteins with a significant FC $<0.667$; black dots, proteins with no significant change. (B) In a heatmap, the upregulation and downregulation of different proteins are observed by cluster analysis. Each line in the figure represents a protein, each column is a sample (C1-4: the asthmatic group, E1-4: the control group), and the colors represent different expression levels (the log 2 value of the quantitative value is obtained and a median correction is carried out during drawing of the heatmap).

A

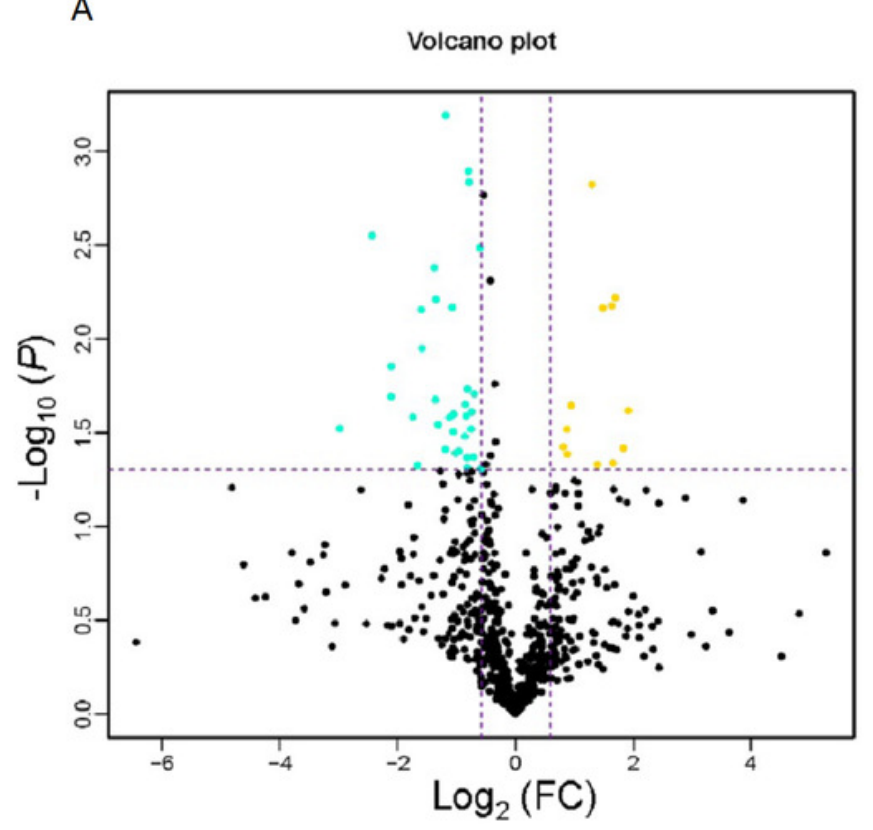

B

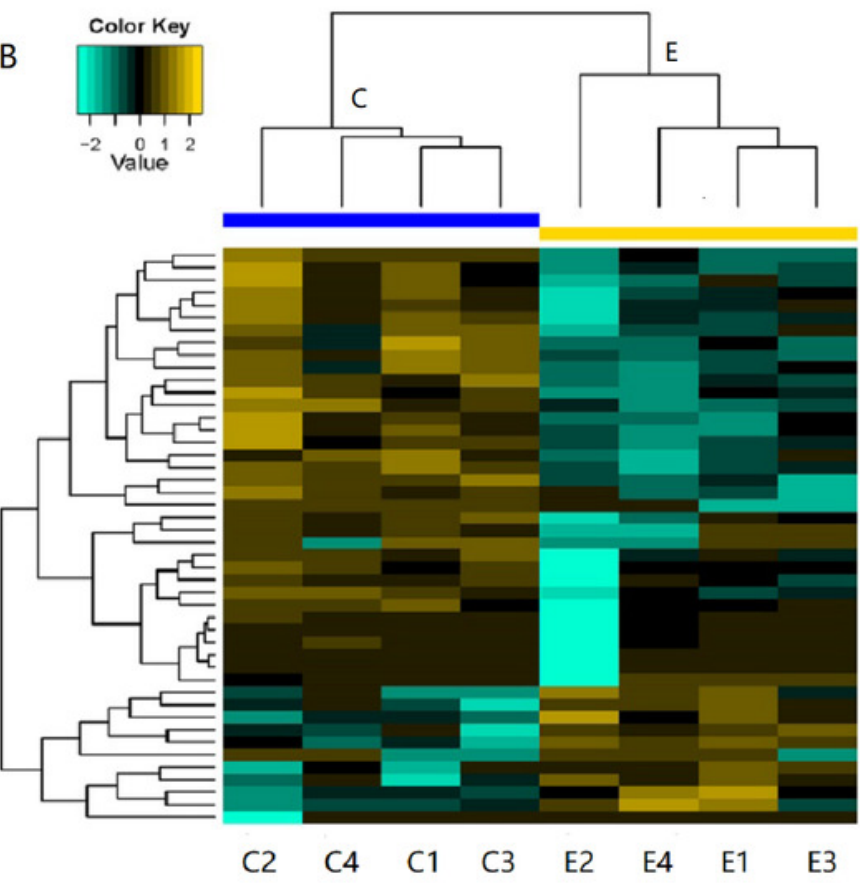


Figure 4

Gene Ontology (GO) annotation for functional classification.

The $\mathrm{x}$-axis represents the classification description (GO term) grouped under each of the three $\mathrm{GO}$ domains listed above the plot, and the $y$-axis represents the number of differentially expressed proteins for each of these terms.

\section{Functional GO Classfication}

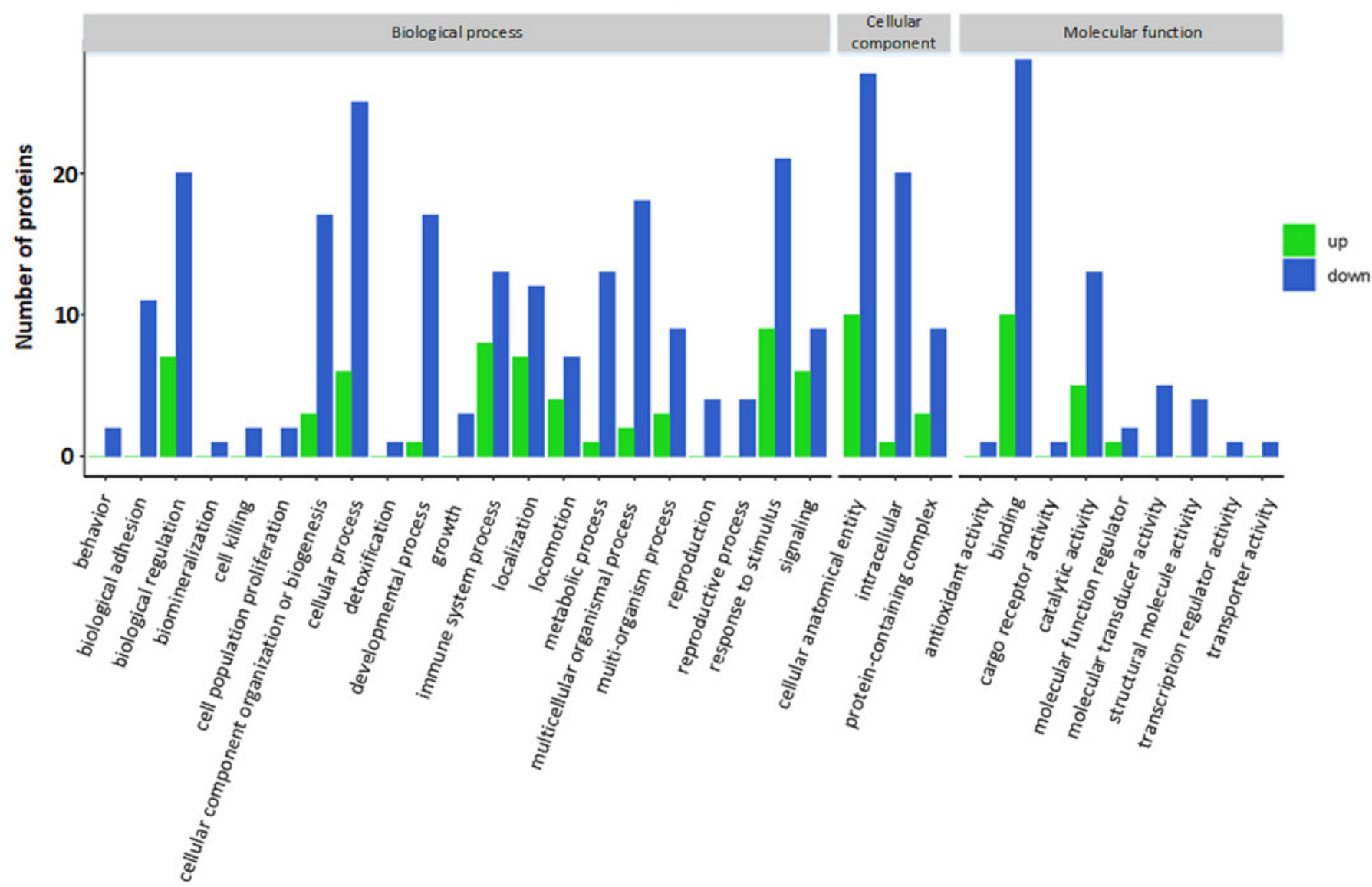




\section{Figure 5}

Gene Ontology (GO) enrichment for functional terms.

The $x$-axis represents the enrichment factor, that is, the proportion of differentially expressed proteins in the total proportion of this $\mathrm{GO}$ classification relative to a multiple of the total proportion of differentially expressed proteins in the $\mathrm{GO}$ classification relative to the proportion of identified proteins in the classification. The y-axis provides the GO term descriptions. The size of the circle represents the number of differentially expressed proteins in the GO term, and the color of the circle indicates the statistical significance of the finding. Fisher exact test $P$ value: $P$ value of the enrichment test obtained using the Fisher exact test; $-\log _{10}(P$ value): $\log$ conversion of the Fisher exact test $P$ value.

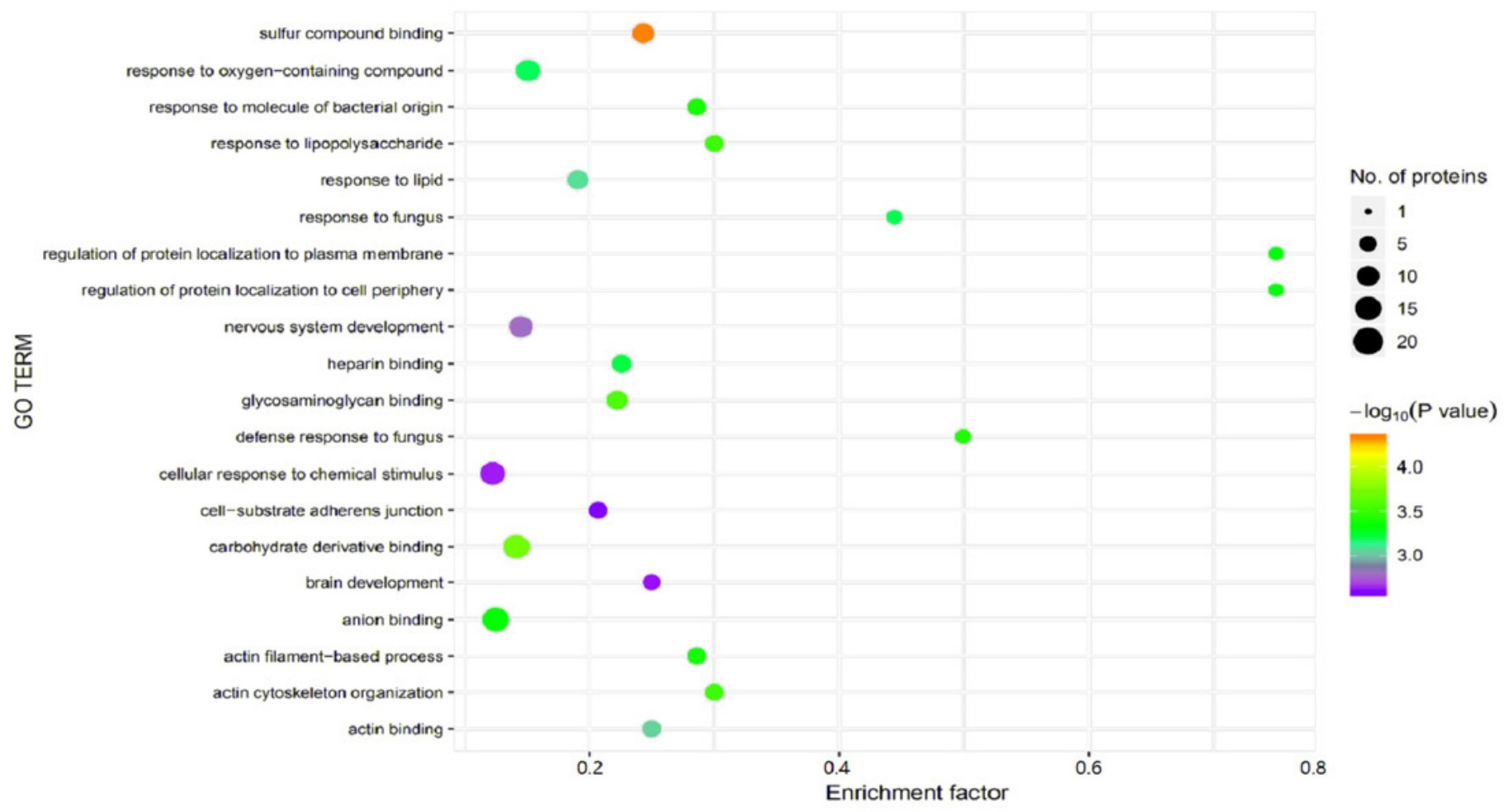




\section{Figure 6}

Kyoto Encyclopedia of Genes and Genomes (KEGG) pathway annotation.

The horizontal axis represents the number of differentially expressed proteins (DEPs), and the vertical axis provides the description of each KEGG pathway. Thus, the number of DEPs identified in this experiment is shown for each specific KEGG signaling pathway annotated for those proteins.

\section{KEGG Pathway}

Dilated cardiomyopathy (DCM) Hypertrophic cardiomyopathy (HCM)

Phagosome

Arrhythmogenic right ventricular cardiomyopathy (ARVC)

Human papillomavirus infection

Regulation of actin cytoskeleton

Focal adhesion

Metabolic pathways

Systemic lupus erythematosus

Proteoglycans in cancer

Shigellosis

Platelet activation

Cell adhesion molecules (CAMs)

ECM-receptor interaction PI3K-Akt signaling pathway

Rap1 signaling pathway

MAPK signaling pathway

Cardiac muscle contraction

Endocytosis

Carbon metabolism

All DEPs shown here are downregulated.

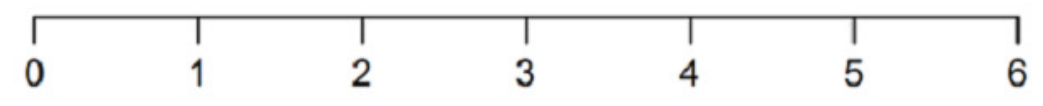

No. of DEPs 


\section{Figure 7}

Kyoto Encyclopedia of Genes and Genomes (KEGG) pathway enrichment.

The horizontal axis represents the enrichment factor, that is, the total proportion of differentially expressed proteins in the KEGG signaling pathway is a multiple of the change in the proportion of identified proteins in the classification. The vertical axis provides a description of the KEGG classification. The size of the circle represents the number of differentially expressed proteins in the KEGG signaling pathway, and the color of the circle indicates the statistical significance of the finding. Fisher exact test $P$ value: $P$ value of the enrichment test obtained using the Fisher exact test; $-\log _{10}(P$ value $)$ : log conversion of the Fisher exact test $P$ value.

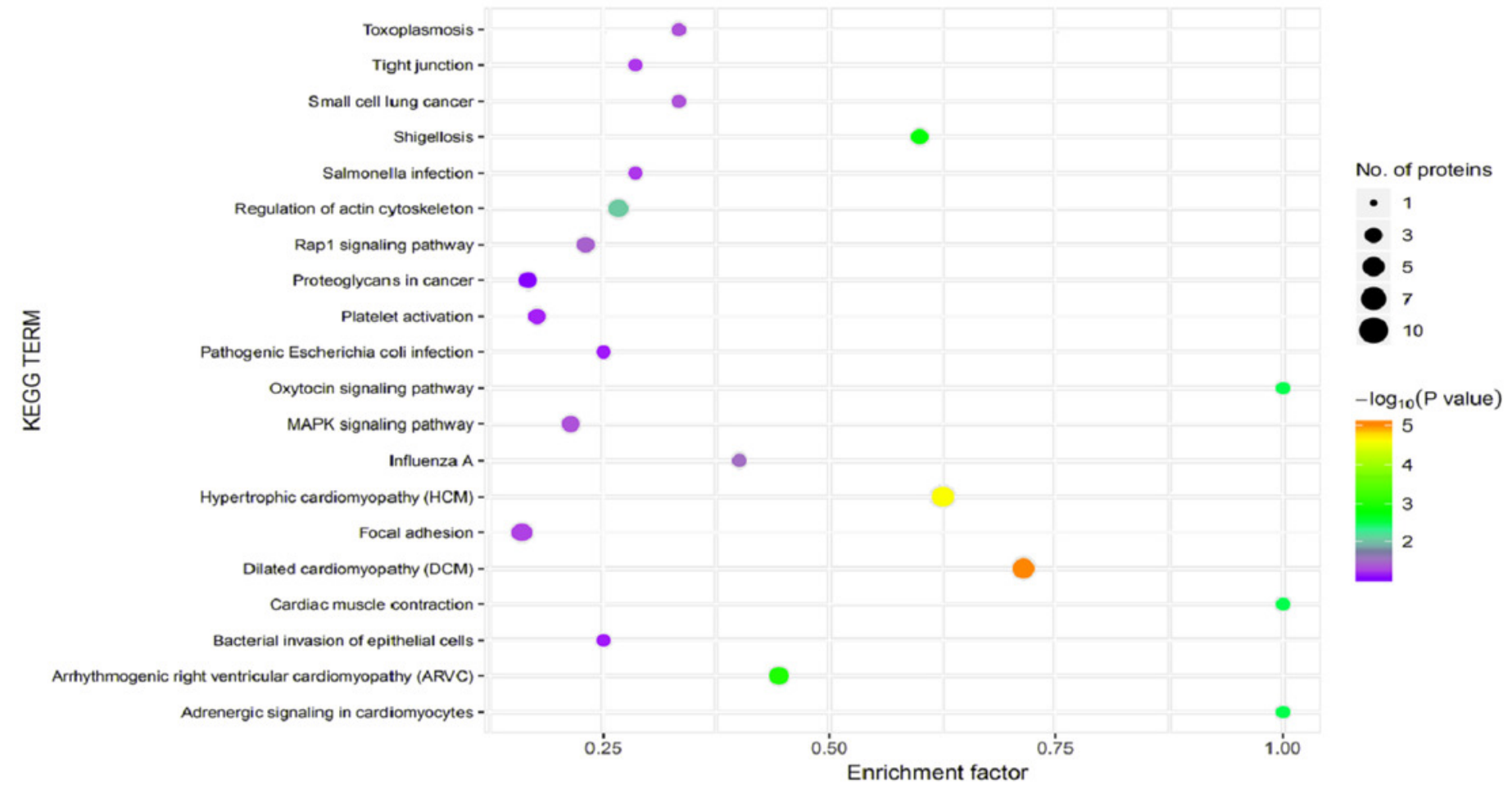


Figure 8

Clusters of Orthologous Groups (COGs) annotated for differentially expressed proteins.

The horizontal axis represents the functional code in the COG database, and the description of that code is shown on the right side of the figure. The $y$-axis indicates the frequency of each functional code.

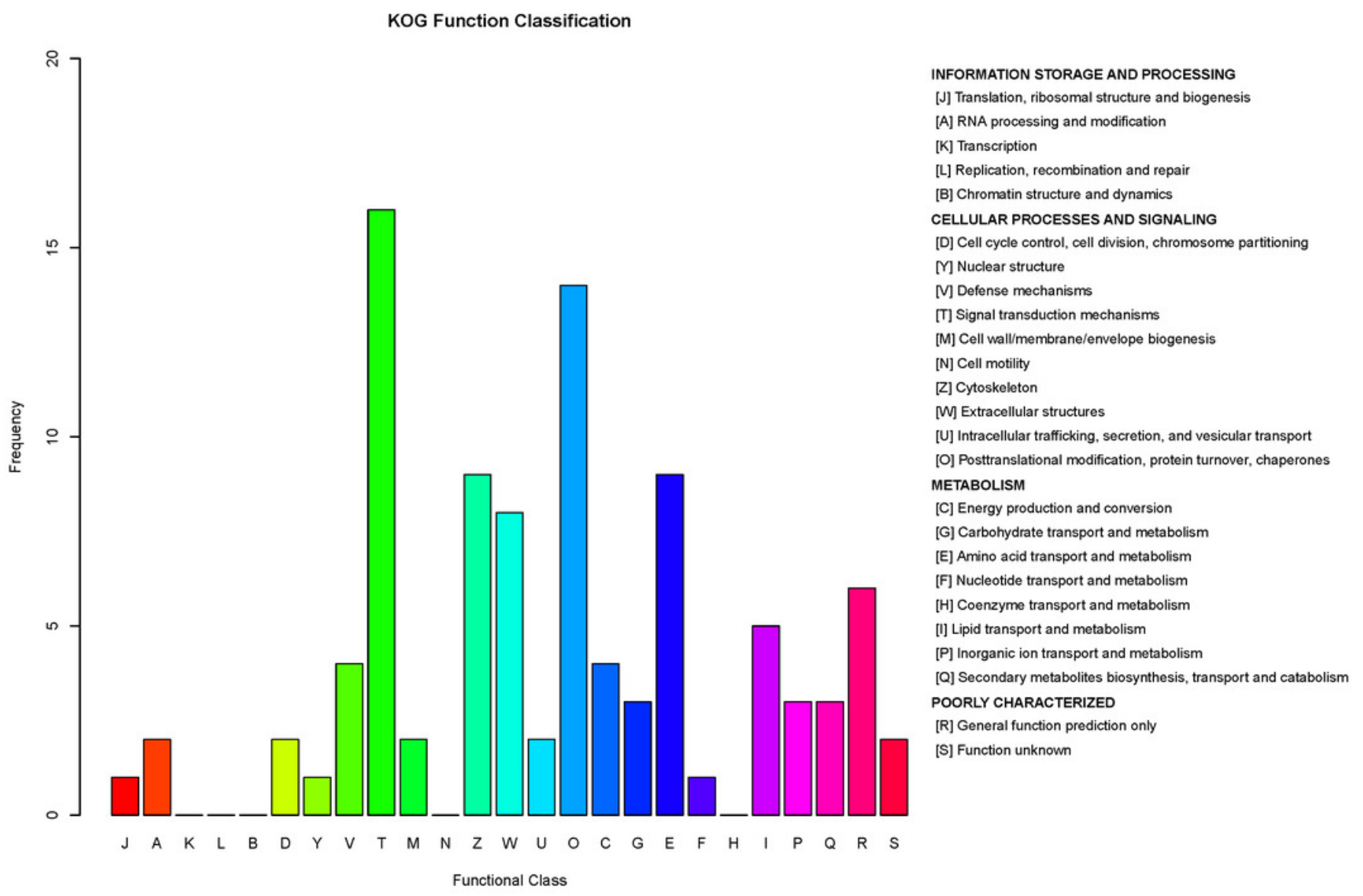


Figure 9

Glucose-6-Phosphate Dehydrogenase (G6PD) protein concentration in serum

The horizontal axis represents the control group and asthma group. The y-axis indicates the concentration of G6PD. The proteins were extracted from the serum of children with or without asthma. Values are shown as the mean \pm SEM $(n=8) ; * P<0.05$ for Control vs. Asthma.

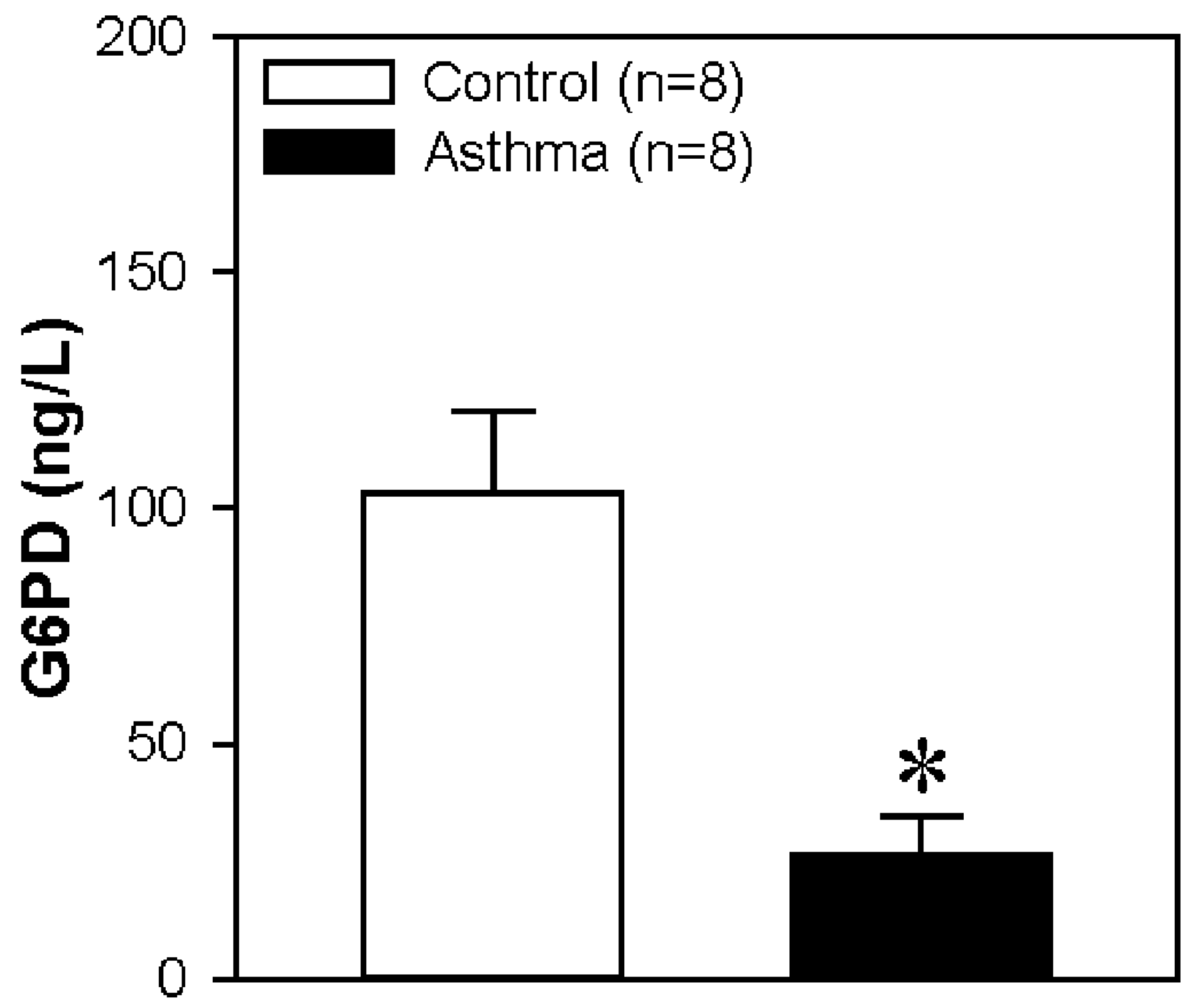




\section{Table 1 (on next page)}

Analysis of Differential Expressed Proteins.

Accession indicates the characteristic numbers of different proteins. Description represents a detailed description of the proteins. 


\section{Table1}

\begin{tabular}{|c|c|c|c|c|c|}
\hline regulated & accession & $\begin{array}{l}\text { gene } \\
\text { name }\end{array}$ & description & $F C$ & $P$ \\
\hline UP & A0A087WW87 & IGKV2-40 & $\begin{array}{l}\text { Immunoglobulin kappa variable } \\
2-40\end{array}$ & 3.75 & 0.024 \\
\hline UP & A0A193CHR8 & - & $\begin{array}{l}\text { 10E8 light chain variable region } \\
\text { (Fragment) }\end{array}$ & 3.55 & 0.038 \\
\hline UP & A2MYE1 & - & A30 (Fragment) & 3.23 & 0.006 \\
\hline UP & А0A0B4J1X5 & IGHV3-74 & $\begin{array}{l}\text { Immunoglobulin heavy variable } 3- \\
74\end{array}$ & 3.14 & 0.046 \\
\hline UP & Q9UL81 & - & $\begin{array}{l}\text { Myosin-reactive immunoglobulin } \\
\text { light chain variable region } \\
\text { (Fragment) }\end{array}$ & 3.1 & 0.007 \\
\hline UP & A0A075B6S5 & IGKV1-27 & $\begin{array}{l}\text { Immunoglobulin kappa variable } 1- \\
27\end{array}$ & 2.79 & 0.007 \\
\hline UP & A2MYD2 & $\mathrm{V} 1-19$ & V1-19 protein (Fragment) & 2.62 & 0.047 \\
\hline UP & A0M8Q6 & IGLC-7 & Immunoglobulin lambda constant 7 & 2.45 & 0.002 \\
\hline UP & A0A218KGR2 & APP & Amyloid beta A4 protein isoform a & 1.93 & 0.023 \\
\hline UP & A2N7P4 & - & $\begin{array}{l}\text { Immunoglobulin mu-chain } \mathrm{D}^{-} \mathrm{J} 4{ }^{-} \\
\text {region (Fragment) }\end{array}$ & 1.84 & 0.041 \\
\hline UP & P04430 & IGKV1-16 & $\begin{array}{l}\text { Immunoglobulin kappa variable } 1^{-} \\
16\end{array}$ & 1.83 & 0.031 \\
\hline $\mathrm{UP}$ & $\mathrm{P} 12273$ & PIP & Prolactin-inducible protein & 1.75 & 0.038 \\
\hline DOWN & C9JF17 & APOD & Apolipoprotein D (Fragment) & 0.67 & 0.049 \\
\hline DOWN & HOYMF1 & ACAN & Aggrecan core protein & 0.66 & 0.003 \\
\hline DOWN & Q12860 & CNTN1 & Contactin-1 & 0.62 & 0.020 \\
\hline DOWN & C9IZP8 & $\mathrm{C} 1 \mathrm{~S}$ & $\begin{array}{l}\text { Complement } \mathrm{C} 1 \mathrm{~s} \text { subcomponent } \\
\text { (Fragment) }\end{array}$ & 0.61 & 0.043 \\
\hline DOWN & Q16853 & AOC3 & Membrane primary amine oxidase & 0.6 & 0.025 \\
\hline DOWN & Q07954 & LRP1 & $\begin{array}{l}\text { Prolow-density lipoprotein } \\
\text { receptor-related protein } 1\end{array}$ & 0.59 & 0.030 \\
\hline DOWN & Q03692 & C0L10A1 & Collagen alpha-1(X) chain & 0.58 & 0.001 \\
\hline DOWN & P05556 & ITGB1 & Integrin beta-1 & 0.58 & 0.001 \\
\hline DOWN & A8K6K4 & - & cDNA FLJ77565, highly similar to & 0.57 & 0.019 \\
\hline
\end{tabular}




\begin{tabular}{|c|c|c|c|c|c|}
\hline & & & $\begin{array}{l}\text { Homo sapiens interleukin } 1 \\
\text { receptor accessory protein } \\
\text { (IL1RAP), transcript variant } 1 \text {, } \\
\text { mRNA }\end{array}$ & & \\
\hline DOWN & P23470 & PTPRG & $\begin{array}{l}\text { Receptor-type tyrosine-protein } \\
\text { phosphatase gamma }\end{array}$ & 0.57 & 0.049 \\
\hline DOWN & Q76LX8 & ADAMTS13 & $\begin{array}{l}\text { A disintegrin and } \\
\text { metalloproteinase with } \\
\text { thrombospondin motifs } 13\end{array}$ & 0.57 & 0.043 \\
\hline DOWN & P27487 & DPP4 & Dipeptidyl peptidase 4 & 0.56 & 0.026 \\
\hline DOWN & P17301 & IFNa 2 & Integrin alpha-2 & 0.55 & 0.022 \\
\hline DOWN & Q59EJ3 & HSPA1A & $\begin{array}{l}\text { Heat shock } 70 \mathrm{kDa} \text { protein } 1 \mathrm{~A} \\
\text { variant (Fragment) }\end{array}$ & 0.55 & 0.033 \\
\hline DOWN & Q59HB3 & APOB & $\begin{array}{l}\text { Apolipoprotein B variant } \\
\text { (Fragment) }\end{array}$ & 0.51 & 0.040 \\
\hline DOWN & B4DWA6 & - & $\begin{array}{l}\text { cDNA FLJ60094, highly similar to } \\
\text { F-actin capping protein subunit } \\
\text { beta }\end{array}$ & 0.5 & 0.041 \\
\hline DOWN & 015394 & NCAM2 & Neural cell adhesion molecule 2 & 0.48 & 0.025 \\
\hline DOWN & A0A3B3ISX9 & TNXB & Tenascin-X & 0.48 & 0.031 \\
\hline DOWN & P60709 & ACTB & Actin, cytoplasmic 1 & 0.47 & 0.007 \\
\hline DOWN & Q9UIU0 & CACNA2D1 & $\begin{array}{l}\text { Dihydropyridine receptor alpha } 2 \\
\text { subunit }\end{array}$ & 0.46 & 0.026 \\
\hline DOWN & P30740 & SERPINB1 & Leukocyte elastase inhibitor & 0.44 & 0.001 \\
\hline DOWN & Q15063 & POSTN & Periostin & 0.44 & 0.039 \\
\hline DOWN & A0A024RAG0 & ALP & Alkaline phosphatase & 0.4 & 0.029 \\
\hline DOWN & A8K3K1 & - & $\begin{array}{l}\text { cDNA FLJ78096, highly similar to } \\
\text { Homo sapiens actin, alpha, } \\
\text { cardiac muscle (ACTC), mRNA }\end{array}$ & 0.39 & 0.006 \\
\hline DOWN & A0A024R5Z9 & PK & Pyruvate kinase & 0.39 & 0.021 \\
\hline DOWN & A0A161I202 & LTF & Lactoferrin & 0.38 & 0.004 \\
\hline DOWN & A0A140GPP7 & - & Prolyl endopeptidase FAP & 0.33 & 0.011 \\
\hline DOWN & Q59G88 & - & Coronin (Fragment) & 0.33 & 0.007 \\
\hline DOWN & P08246 & ELANE & Neutrophil elastase & 0.32 & 0.047 \\
\hline DOWN & P08311 & CTSG & Cathepsin G & 0.3 & 0.026 \\
\hline DOWN & P05164 & MP0 & Myeloperoxidase & 0.23 & 0.014 \\
\hline
\end{tabular}




\begin{tabular}{|c|c|c|c|c|c|}
\hline DOWN & B2RDY9 & - & $\begin{array}{l}\text { Adenylyl cyclase-associated } \\
\text { protein }\end{array}$ & 0.23 & 0.020 \\
\hline DOWN & A8K8D9 & G6PD & $\begin{array}{l}\text { Glucose-6-phosphate }{ }^{-} \text {- } \\
\text { dehydrogenase }\end{array}$ & 0.19 & 0.003 \\
\hline DOWN & P07737 & PFN1 & Profilin-1 & 0.13 & 0.030 \\
\hline
\end{tabular}

2 